Artigo

\title{
Personalidade e Padrões Comportamentais do Sono: Diferenças entre Homens e Mulheres
}

\author{
Victor Hugo Dias Pereira \\ Orcid.org/0000-0001-6942-7369 \\ Luiz Henrique de Carvalho Diniz Melo \\ Orcid.org/0000-0002-4461-6267 \\ Natanael Antonio dos Santos \\ Orcid.org/0000-0001-7708-9929 \\ Melyssa Kellyane Cavalcanti Galdino \\ Orcid.org/0000-0001-7180-3458 \\ Michael Jackson Oliveira Andrade* \\ Orcid.org/0000-0002-2650-451X
}

Universidade Federal da Paraíba, João Pessoa, PB, Brasil

\begin{abstract}
Resumo
O presente estudo teve o objetivo de verificar os efeitos comportamentais do sono nos traços de personalidade de adultos jovens. Participaram desta pesquisa 114 voluntários entre 18 e 40 anos de idade. Os sujeitos foram divididos em três grupos: Moderadamente Matutino $(n=23)$; Intermediário $(n=52) \mathrm{e}$ Moderadamente Vespertino $(n=39)$. Entre os instrumentos de pesquisa utilizou-se o Questionário de Matutinidade e Vespertinidade de Horne e Östberg e Inventário dos Cinco Grandes Fatores de Personalidade. A MANOVA mostrou diferença significativa entre os cronotipos e os traços de personalidade $\left[\lambda=0,78 ; F(10,198)=2,52 ; p<0,05 ; \eta^{2}=0,11\right]$. Observou-se diferença significativa apenas para os traços de extroversão $\left[F(2,103)=3,65 ; p<0,05 ; \eta^{2}=0,06\right]$ e amabilidade $[F(2,103)=8,03 ; p<0,05$; $\left.\eta^{2}=0,14\right]$. Este estudo sugere que pessoas com diferentes traços de personalidades possuem horas específicas para realizar suas atividades e que existe uma relação com o padrão do cronotipo. Em geral, os dados indicam que homens e mulheres apresentam traços de personalidade diferentes em relação aos efeitos comportamentais do sono.
\end{abstract}

Palavras-chave: Cronotipo, sono, traços de personalidade.

\section{Personality Traits and Behavioral Sleep Patterns: Differences between Men and Women}

\begin{abstract}
This study aimed to investigate the behavioral effects of sleep on the personality traits of young adults. The sample consisted of 114 volunteers with ages between 18 and 40 . The subjects were characterized

* Endereço para correspondência: Universidade Federal da Paraíba, Centro de Ciências Humanas, Letras e Artes, Departamento de Pós-graduação em Psicologia Social, Cidade Universitária, João Pessoa, PB, Brasil 58051900. Fone: 55 (83) 3216-7200, 55 (83) 3216-7064. E-mail: m.jackson_20@yahoo.com.br, victorhdiaspereira@ gmail.com,lhenrique_melo@hotmail.com,natanael_labv@yahoo.com.bre melyssa_cavalcanti@hotmail.com Financiamento: Universidade Federal da Paraíba.
\end{abstract}


into three groups according to Horne and Östberg (1975): Moderately Morning ( $n=23)$; intermediary $(n=52)$ and Moderately Evening $(n=39)$. The Horne and Östberg Morningness and Eveningness questionnaire and the Big Five Personality Inventory were used for data collection. MANOVA showed a significant difference between the chronotype and personality traits $[\lambda=0.78 ; F(10.198)=2.52 ; p<$ $\left..05 ; \eta^{2}=0.11\right]$. The results showed a significant difference on extraversion $\left[F(2.103)=3.65 ; p<.05 ; \eta^{2}\right.$ $=0.06]$ and kindness traits $\left[F(2.103)=8.03 ; p<.05 ; \eta^{2}=0.14\right]$. The investigation suggests that among these subjects, different personality traits may influence preference for certain periods of the day to carry out daily activities and that there is a relationship with the standard chronotype. Also, the results indicate that men and women have different personality traits when the effects of the sleep behavior are checked.

Keywords: Chronotype, sleep, personality traits.

\section{Rasgos de Personalidad y lós Patrones de Sueño de Comportamiento: Diferencias entre Hombres y Mujeres}

\section{Resumen}

Este estúdio tuvo como objetivo verificar los efectos del comportamento del sueño em los rasgos de personalidad de los adultos jóvenes. El estúdio recogió 114 voluntarios de entre 18 y 40 años de edad. Los sujetos fueron divididos entres grupos: Moderadamente mañana $(n=23)$; Intermedio $(n=52)$ y moderadamente Vespertino $(n=39)$. Entre el instrumento de estudio utilizado el Cuestionario matutinidad y vespertinidad Horne y Östberg y el Inventario de los cinco grandes factores. MANOVA mostró una diferencia significativa entre el cronotipo y rasgos de personalidad $[\lambda=0.78 ; F(10.198)=2.52 ; p<.05$; $\left.\eta^{2}=0.11\right]$. Los resultados mostraron una diferencia significativa en la extraversión $[F(2.103)=3.65 ; p$ $\left.<.05 ; \eta^{2}=0.06\right]$ y rasgos de bondad $\left[F(2.103)=8.03 ; p<.05 ; \eta^{2}=0.14\right]$. La investigación sugiere que entre estos sujetos, diferentes rasgos de personalidad pueden influir en la preferencia por ciertos períodos del día para llevar a cabo las actividades diarias y que existe una relación con el cronotipo estándar. Además, los resultados indican que hombres y mujeres tienen diferentes rasgos de personalidad cuando se comprueban los efectos del comportamiento del sueño.

Palabras clave: Cronotipo, sueño, rasgos de personalidade.

A Cronobiologia possui a função de estudar a variação dos ritmos biológicos. É possível demonstrar que vários processos cerebrais apresentam um padrão regular com ritmo aproximado de 24 horas (Blatter \& Cajochen, 2007; Marques $\&$ Menna-Barreto, 1997). Os seres humanos recebem influências externas periodicamente ao longo do dia com a função de organizar informações no sistema nervoso central (Valdez, Ramírez, \& Garcia, 2012). Intrínseco a estes padrões rítmicos, os processos cognitivos e comportamentais admitem funções temporais sincronizadas entre o meio interno e externo do organismo (Blatter \& Cajochen, 2007). Por exemplo, o sono, um estado comportamental bem definido em arquiteturas de fases eletroencefalográficas, desempenha um papel fundamental para sincro- nização neurocognitiva (Besílio, Carneiro, Silva, Fortes, \& Araújo, 2012).

A tipologia circadiana ou padrão do cronotipo tem sido associado a funções cognitivas e comportamentais de processos psicológicos, como por exemplo, medidas atencionais, mnemônicas, tomadas de decisão e percepção social (Tankova, Adan, \& Buela-Casal, 1994). De forma geral, pessoas matutinas, ou seja, que possuem preferência para realizar atividades no período da manhã, e pessoas vespertinas, as quais possuem preferência em realizar atividades no turno da noite, associam níveis de excitação cerebral conforme perfis de traços de personalidade. Em particular, as variações rítmicas interindividuais demonstram domínios específicos de traços de personalidade com preferência ma- 
tutina ou vespertina (DeYoung, Hasher, Djikic, Criger, \& Peterson, 2007; Larsen, 1985; Mecacci, Zani, Rocchetti, \& Lucioli, 1986, Mitchell \& Redman, 1993; Neubauer, 1992; Tonetti et al., 2010; Torsvall \& Åkerstedt, 1980).

Diferentes estudos mostram que sujeitos vespertinos tendem a apresentar maior propensão para traços de extroversão, principalmente quando o traço de personalidade está associado a padrões de sociabilidade (Larsen, 1985; Mecacci et al., 1986; Randler, Baumann, \& Horzum, 2014). DeYoung et al. (2007) e Tonetti et al. (2010) mostraram ainda que além da extroversão, indivíduos vespertinos podem apresentar comportamentos característicos de neuroticismo e psicotismo, sendo menos dependentes que sujeitos matutinos. Por outro lado, os sujeitos matutinos tendem a ser mais introvertidos e apresentar maior envolvimento a valores socioculturais (Russo, Leone, Penolazzi, \& Natale, 2012).

Em contrapartida, o estudo de Neubauer (1992) sugere que extroversão, também associado a componente de impulsividade, correlaciona-se positivamente a padrões de matutinidade. Além disso, a investigação indicou uma intrínseca associação entre neuroticismo e vespertinidade. Esses achados sugerem uma associação entre traço de personalidade com padrão de cronotipo. Já Horne e Östberg (1975) mostraram que sujeitos com altos escores no traço extroversão tendem a realizar suas atividades no período noturno, enquanto sujeitos com altos escores em introversão demostram preferências por atividades no período da manhã. Apesar dos estudos apresentamrem correlação de dados para sujeitos adultos, as pesquisas ainda são contraditórias e não apresentam resultados conclusivos.

Randler (2007) procurou associar o cronotipo com os traços de personalidade utilizando o modelo hierárquico dos Cinco Grandes Fatores de Personalidade. De acordo com John e Srivastava (1999), este modelo é derivado de análises dos termos da linguagem que os indivíduos usam em seus ambientes naturais para descrever a si próprios e os outros. A validação desta escala no Brasil apresenta cinco categorias de personalidade: abertura, conscienciosidade, extroversão, amabilidade e neuroticismo (Andrade, 2008). Randler (2007) aponta que homens e mulheres matutinos correlacionam-se positivamente com traços de amabilidade, conscienciosidade e socialização, corroborando os resultados de Jackson e Gerard (1996). Ainda, Randler e Saliger (2011) mostraram que adolescentes matutinos apresentam maiores escores em temperamentos de persistência e cooperação, enquanto os adolescentes vespertinos apresentam maiores escores em busca por novidades e estão propensos a dependência por recompensa. De forma geral, os pesquisadores atribuíram esses resultados a uma função excitatória dos neurotransmissores em tempo circadianos, porém não explicam como estes mecanismos neurais se ajustam a presença e ausência de luz (principal sincronizador circadiano).

Segundo Roenneberg et al. (2004) os fatores como idade e sexo podem influenciar na construção da arquitetura do sono e da personalidade. Por exemplo, o atraso do início da fase do sono é diferente entre homens e mulheres. Para os homens acontece por volta dos 21 anos de idade já nas mulheres o comportamento antecede dois anos, ou seja, aos 19 anos de idade. A idade apresenta uma funcionalidade biológica distinta para ambos os sexos e proporciona uma maturação na preferência pelo cronotipo (Díaz-Morales, de León, \& Sorroche, 2007; Randler, 2007). Ainda, a diferença na velocidade de desenvolvimento puberal entre meninos e meninas é um fator determinante nas mudanças que ocorrem nos ritmos circadianos (Díaz-Morales et al., 2007). Assim, aspectos do sono podem estar associados a percepções de saúde, níveis de energia, bem-estar, status funcional e medidas de traços de personalidade em homens e mulheres (Duggan, Friedman, McDevitt, \& Mednick, 2014). O presente estudo parte da hipótese que influências e experiências da vida social de adultos jovens diferenciam-se quanto ao seu cronotipo, ou seja, a formação de traços de personalidade de homens e mulheres é diferente conforme seu padrão por matutinidade e vespertinidade (Randler, 2007).

Como visto a relação entre o ciclo sono-vigília e os níveis de qualidade do sono podem modificar o funcionamento cognitivo e compor- 
tamental dos sujeitos, influenciando indiretamente nos traços de personalidade (Randler et al., 2014). As diferenças encontradas nos traços de personalidade associadas ao cronotipo propõem perspectivas cronopsicológicas visando uma construção de um modelo teórico que explique causas e manifestações tipológicas circadianas relacionais aos traços de personalidade.

Ainda que uma quantidade expressiva de estudos quantifique a relação entre cronotipo e traços de personalidade, observam-se uma grande quantidade de resultados inconclusivos e mal comprendidos, como também lacunas na investigação dos efeitos do comportamento do sono em traços de personalidades de homens e mulheres. Dessa forma, o presente estudo teve como objetivo principal verificar os efeitos comportamentais do sono nos traços de personalidade de homens e mulheres adultos jovens.

\section{Método}

\section{Sujeitos}

Participaram desta pesquisa 114 voluntários entre 18 e 40 anos de idade. Os sujeitos foram divididos em três grupos de acordo com o padrão de cronotipo (Tabela 1). Inicialmente foram retirados 45 participantes da pesquisa por apresentarem índices de ansiedade leve (75,5\%) e depressão moderada (25,5\%). Todos os voluntários eram universitários e encontravam-se clinicamente estáveis, não apresentando transtornos neuropsiquiátricos identificáveis, estando livres de qualquer transtorno que afetasse o sono. Também foi excluído do estudo qualquer participante que fizesse uso de algum tipo de fármaco que afetasse o sistema nervoso central ou substâncias tóxicas.

Tabela 1

Dados da Amostra por Padrão de Comportamento do Sono ou Cronotipo

\begin{tabular}{|c|c|c|c|c|}
\hline \multirow{2}{*}{ Cronotipo } & \multirow{2}{*}{$\begin{array}{c}\text { Homem } \\
n\end{array}$} & \multicolumn{3}{|c|}{ Mulher } \\
\hline & & Idade & $n$ & Idade \\
\hline Moderadamente Matutinos & 7 & $24,86(6,9)$ & 16 & $22,06(3,4)$ \\
\hline Intermediário & 13 & $22,23(3,6)$ & 39 & $20,72(3,6)$ \\
\hline Moderadamente Vespertinos & 13 & $24,69(3,1)$ & 26 & $21,12(3,9)$ \\
\hline Total & 33 & $23,93(4,5)$ & 78 & $21,3(3,6)$ \\
\hline
\end{tabular}

\section{Instrumentos}

Questionário Sociodemográfico. Este questionário foi composto por questões sociais (como sexo, idade, preferência de horário, renda mensal, entre outras) e clínicas (atividades físicas, comprometimentos psicológicos e neurológicos, consumo de substâncias psicoativas, entre outras) de acordo com as necessidades do estudo.

Questionário de Matutinidade e Vespertinidade de Horne e Östberg. Elaborado por Horne e Östberg (1975) e adaptado/validado para a língua portuguesa por Benedito-Silva, Menna-Barreto, Marques e Tenreiro (1990). O instrumento tem como finalidade avaliar a preferência do indivíduo em realizar suas atividades durante o período de 24 horas. Composto por 19 ques- tões acerca de situações habituais do cotidiano do indivíduo e seus respectivos escores, classificam os indivíduos em cinco categorias de acordo com o cronotipo: vespertino (16 a 30 pontos); moderadamente vespertino ( 31 a 41 pontos); indiferente ou intermediário (42 a 58 pontos); moderadamente matutino (59 a 69 pontos); e matutino (70 a 86 pontos).

Índice de Qualidade do Sono de Pittsburgh (PSQI). O instrumento consiste em 19 questões e é dividido em sete componentes subjetivos sobre o sono em relação ao último mês: 1) qualidade do sono; 2) latência do sono; 3) duração do sono; 4) eficiência habitual do sono; 5) distúrbios do sono; 6) uso de medicamentos para dormir; 7) sonolência diurna e distúrbios durante todo o 
dia. Cada componente possui alternativas com pontuações que variam entre zero e 3 pontos, onde zero indica nenhum e 3 aponta problema grave, ou seja, qualidade de sono pobre. A soma total dos pontos varia de 0 a 21 pontos (Furlani $\&$ Ceolim, 2005). Destaca-se que sua validação em português foi realizada com alto índice de sensibilidade (65\%).

Diário do Sono. Este instrumento foi elaborado pelo próprio laboratório e seu preenchimento visou descrever padrões de comportamento do sono durante um período de 14 dias consecutivos conforme os itens: latência de sono, eficiência do sono, a forma de despertar e os horários e duração de cochilos.

Inventário dos Cinco Grandes Fatores de Personalidade (IGFP-5). O IGPF-5 possui 44 itens, estruturados em uma escala do tipo Likert que varia de descordo totalmente (1) até concordo totalmente (5). Os itens encontram-se agrupados em cinco fatores: Abertura, Conscienciosidade, Extroversão, Amabilidade e Neuroticismo. Sua validação para população brasileira foi realizada por Andrade (2008) em uma amostra de 5.089 com faixa etária de 13 a 67 anos.

Inventário de Ansiedade de Beck (BAI). Possui 21 itens, avalia a intensidade da ansiedade clínica $(\alpha=0,92)$. Segundo Cunha (1999), a interpretação dos níveis de ansiedade pode ser vista da seguinte maneira: limite mínimo (0-10 pontos); ansiedade leve (11-19 pontos); ansiedade moderada (20-30 pontos); e ansiedade severa (31-63 pontos).

Inventário de Depressão de Beck (BDI). Esse inventário é composto por 21 categorias de sintomas e atitudes que descrevem manifestações comportamentais, cognitivas, afetivas e somáticas da depressão (Cunha, 1999). Cada categoria contém de quatro a cinco alternativas que expressam níveis de gravidade dos sintomas depressivos. A pontuação para cada categoria varia de zero a 3 , sendo o menor valor a ausência dos sintomas depressivos e o maior valor a presença dos sintomas mais intensos. A interpretação dos resultados é subdividida em: ausência de depressão (até 9 pontos); depressão leve a moderada (10-18 pontos); depressão moderada a grave (19-29); e depressão grave (30-63 pontos).
Escala Analógica Visual para Atenção. Elaborada para avaliar aspectos de experiências subjetivas relacionadas à fadiga, tensão, atenção e estado de alerta do participante durante um período de 24 horas. O participante é indicado a assinalar em uma escala de zero a 20 como se sente no presente momento em relação a cada uma das experiências subjetivas indicadas. Nesta escala o zero corresponde a Nada e 20 a Muito (Laneiro, Brites, Tap, Silva, Reguinga, \& Guerra, 2011).

\section{Procedimento}

O presente estudo foi realizado na Universidade Federal da Paraíba. Inicialmente foi realizado o contato com os participantes para explicar sobre questões éticas e procedimentos a ser realizado na pesquisa, esse passo foi feito em sala de aula com o consentimento esclarecido dos professores. Nesse momento, foram entregues aos participantes os questionários de levantamento amostral (Questionário Sóciodemográfico, Questionário de Matutinidade e Vespertinidade de Horne \& Östberg, Inventário dos Cinco Grandes Fatores de Personalidade [IGFP5], Índice de qualidade do sono de Pittsburgh [PSQI], Inventário de Ansiedade de Beck [BAI], e o Inventário de Depressão de Beck, BDI). Conforme as explicações, os participantes responderam os questionários de maneira individual. Todos os instrumentos eram auto aplicáveis.

Após responderem os questionários de levantamento amostral, os participantes receberam a Escala Analógica Visual para Atenção e o Diário do Sono. Todos foram instruídos a responder as escalas por um período de 14 dias consecutivos, iniciando sempre em uma sexta-feira (dia que eram entregues os protocolos), a fim de compreender um padrão de comportamento em dias úteis e não úteis da semana. As quatro dimensões da Escala Analógica Visual para Atenção foram respondidas diariamente nos horários das $9 \mathrm{~h}, 15 \mathrm{~h}$ e 21 horas. Da mesma maneira, o Diário do Sono foi respondido diariamente com conteúdo sobre hábitos sociais diurnos e noturnos. Foi recomendado aos participantes que se mantivessem assíduos em suas respostas diárias e que fossem feitas preferencialmente no período da noite. 
Após a entrega do material, as respostas dos participantes foram plotadas em planilhas de acordo com o cronotipo e as variáveis de interesse, sendo realizadas análises estatísticas descritivas e inferenciais por meio do SPSS (Statistical Package for the Social Sciences), versão 20. As análises descritivas dos dados foram verificadas através de frequências e medidas de tendência central. As condições de normalidade multivariada dos dados foram verificadas antes da aplicação dos testes estatísticos inferenciais dentro do intervalo de confiança de 95\% (Komogorov-Sminorv). Utilizou-se análise de variância multivariada (MANOVA one way) com estatística Lambda de Wilks para avaliar a hipótese do estudo. Consequentemente foi verificada a comparação múltipla dos fatores através da ANOVA. Depois de identificado a significância entre as ANOVA utilizou-se o teste post-hoc Tukey e Bonferroni para verificar as comparações múltiplas das médias.

\section{Aspectos Éticos}

O estudo foi submetido na base unificada de registros de pesquisas envolvendo seres humanos (Plataforma Brasil) e aprovado pelo Comitê de Ética do Centro de Ciências da Saúde (CCS) da instituição pública filiada sob o protocolo $n^{\circ} .32183114 .7 .0000 .5183$. A participação na pesquisa foi voluntária, sendo garantido o anonimato e o sigilo das informações mediante a assinatura do Termo de Consentimento Livre e Esclarecido (TCLE), seguindo os preceitos da Resolução $n^{\circ}$. 466/12 do Conselho Nacional de Saúde.

\section{Resultados}

\section{Aspectos Gerais}

Inicialmente não foi observada diferença significativa entre as idades e o nível de qualidade de sono entre os participantes de acordo com o cronotipo $(p>0,01)$. A maior parte dos participantes era do sexo feminino (77\%), destes $48 \%$ possuíam cronotipo do tipo Intermediário. A análise também apontou que não houve diferença entre os grupos em relação ao consumo de drogas como o café e o álcool $(p>0,01)$. Todos os participantes estavam livres de doenças relacionadas ao sono, porém $5 \%$ possuíam algum parente de terceiro grau com algum transtorno neuropsiquiátrico.

\section{Dimensões Comportamentais do Sono}

A análise de variância (ANOVA one way) mostrou diferença significativa durante a hora de deitar e de acordar entre os cronotipos (Tabela 2). Os dados mostraram que durante os dias úteis da semana os sujeitos apresentam diferenças para hora de deitar $[\mathrm{F}(2,100)=3,20 ; p<0,05]$ e hora de acordar $[F(2,100)=9,41 ; p<0,05]$. $\mathrm{O}$ teste post hoc Tukey mostrou que os sujeitos MV procuram dormir mais tarde e acordar mais tarde do que os sujeitos MM $(p<0,05)$. Esses padrões de comportamentos do sono se mantiveram durante os finais de semana tanto para hora de dormir $[F(2,106)=2,72 ; p<0,05]$ e o hora de acordar $[F(2,104)=27,75 ; p<0,05]$. Novamente, os sujeitos MV foram dormir mais tarde que os sujeitos I e MM $(p<0,05)$. Além disso, os sujeitos Intermediários foram dormir mais tarde que os sujeitos MM $(p<0,05)$. Essas medidas se repetiram entre os sujeitos em relação à hora de acordar $(p<0,05)$. Não houve diferenças nas latências entre os minutos para hora de acordar e dormir entre os grupos, independentemente dos dias da semana.

Os resultados também apontaram diferenças significativas entre os cronotipos e as dimensões da escala analógica visual de acordo com as dimensões avaliadas (Tabela 3). Para dimensão fadiga: houve diferença no período das $21 \mathrm{~h}$ $[F(2,39)=3,28 ; p<0,05]$ sendo que sujeitos I apresentaram maior fadiga do que os MM ( $p<$ $0,05)$. Em relação às dimensões atenção e estado de alerta os dados mostraram que: os sujeitos apresentaram diferenças significativas no horário das $15 \mathrm{~h}$ tanto para atenção $[F(2,39)=4,63$; $p<0,05]$ quanto para o estado de alerta $[F(2,39)$ $=7,37 ; p<0,05]$. De forma geral, os participantes MV apresentaram maiores níveis de atenção e estado de alerta do que os sujeitos MM ( $p<$ $0,05)$, além de apresentarem maiores níveis de alerta do que os sujeitos I $(p<0,05)$. Os dados não apresentaram diferença significativa para a dimensão sonolência. 
Tabela 2

Padrões Comportamentais do Sono de acordo com o Diário do Sono nos Dias de Semana e Finais de Semana

\begin{tabular}{|c|c|c|c|c|c|c|}
\hline \multirow{2}{*}{ Diário do Sono } & \multicolumn{2}{|c|}{$\begin{array}{l}\text { Moderadamente } \\
\text { Matutino }\end{array}$} & \multicolumn{2}{|c|}{ Intermediário } & \multicolumn{2}{|c|}{$\begin{array}{c}\text { Moderadamente } \\
\text { Vespertino }\end{array}$} \\
\hline & $M$ & $D P$ & $M$ & $D P$ & $M$ & $D P$ \\
\hline \multicolumn{7}{|l|}{ Semana } \\
\hline Hora de Dormir & $00: 39$ & \pm 178 & $23: 50$ & \pm 94 & $23: 11$ & \pm 85 \\
\hline Latência de Deitar & 12 & \pm 17 & 11 & \pm 11 & 15 & \pm 18 \\
\hline Hora de Acordar & $07: 48$ & \pm 113 & 07:09 & \pm 105 & $07: 48$ & \pm 113 \\
\hline Latência de Acordar & 12 & \pm 17 & 11 & \pm 11 & 15 & \pm 18 \\
\hline \multicolumn{7}{|l|}{ Fim de Semana } \\
\hline Hora de Dormir & $01: 20$ & \pm 131 & $00: 18$ & \pm 88 & $23: 44$ & \pm 111 \\
\hline Latência de Deitar & 20 & \pm 24 & 14 & \pm 16 & 18 & \pm 18 \\
\hline Hora de Acordar & 09:40 & \pm 111 & $08: 19$ & \pm 92 & $09: 40$ & \pm 111 \\
\hline Latência de Acordar & 20 & \pm 24 & 14 & \pm 16 & 18 & \pm 18 \\
\hline
\end{tabular}

Tabela 3

Escores da Escala Analógica Visual de acordo com o Cronotipo e o Horário de Medida

\begin{tabular}{|c|c|c|c|c|c|c|c|c|c|}
\hline \multirow{2}{*}{$\begin{array}{c}\text { Escala } \\
\text { Analógica } \\
\text { Visual }\end{array}$} & \multicolumn{3}{|c|}{ Moderadamente Matutino } & \multicolumn{3}{|c|}{ Intermediário } & \multicolumn{3}{|c|}{ Moderadamente Vespertino } \\
\hline & $9 \mathrm{~h}$ & $15 \mathrm{~h}$ & $21 \mathrm{~h}$ & $9 \mathrm{~h}$ & $15 \mathrm{~h}$ & $21 \mathrm{~h}$ & $9 \mathrm{~h}$ & $15 \mathrm{~h}$ & $21 \mathrm{~h}$ \\
\hline \multicolumn{10}{|l|}{ Semana } \\
\hline Fadiga & $\begin{array}{l}5,51 \\
(5,1)\end{array}$ & $\begin{array}{c}7,57 \\
(5,5)\end{array}$ & $\begin{array}{c}10,46 \\
(6,0)\end{array}$ & $\begin{array}{l}7,37 \\
(5,2)\end{array}$ & $\begin{array}{l}8,28 \\
(4,7)\end{array}$ & $\begin{array}{l}9,98 \\
(5,3)\end{array}$ & $\begin{array}{c}10,34 \\
(5,7)\end{array}$ & $\begin{array}{l}8,92 \\
(5,8)\end{array}$ & $\begin{array}{l}8,82 \\
(5,8)\end{array}$ \\
\hline Sonolência & $\begin{array}{l}5,57 \\
(4,9)\end{array}$ & $\begin{array}{l}7,29 \\
(5,4)\end{array}$ & $\begin{array}{l}11,32 \\
(6,5)\end{array}$ & $\begin{array}{l}8,35 \\
(5,4)\end{array}$ & $\begin{array}{l}8,72 \\
(4,9)\end{array}$ & $\begin{array}{c}10,34 \\
(5,1)\end{array}$ & $\begin{array}{c}12,02 \\
(5,2)\end{array}$ & $\begin{array}{l}9,97 \\
(5,6)\end{array}$ & $\begin{array}{l}9,80 \\
(5,7)\end{array}$ \\
\hline Atenção & $\begin{array}{c}12,09 \\
(5,7)\end{array}$ & $\begin{array}{l}11,29 \\
(5,3)\end{array}$ & $\begin{array}{l}9,70 \\
(5,7)\end{array}$ & $\begin{array}{c}10,69 \\
(4,9)\end{array}$ & $\begin{array}{l}11,55 \\
(4,1)\end{array}$ & $\begin{array}{c}10,91 \\
(4,4)\end{array}$ & $\begin{array}{l}9,35 \\
(5,0)\end{array}$ & $\begin{array}{c}11,74 \\
(4,7)\end{array}$ & $\begin{array}{l}12,05 \\
(4,5)\end{array}$ \\
\hline Estado de Alerta & $\begin{array}{l}9,43 \\
(5,1)\end{array}$ & $\begin{array}{l}11,62 \\
(4,9)\end{array}$ & $\begin{array}{l}12,22 \\
(4,5)\end{array}$ & $\begin{array}{l}10,68 \\
(5,2)\end{array}$ & $\begin{array}{l}11,50 \\
(4,8)\end{array}$ & $\begin{array}{c}10,72 \\
(4,6)\end{array}$ & $\begin{array}{l}9,43 \\
(5,1)\end{array}$ & $\begin{array}{l}11,62 \\
(4,9)\end{array}$ & $\begin{array}{c}12,22 \\
(4,5)\end{array}$ \\
\hline \multicolumn{10}{|l|}{ Fim de Semana } \\
\hline Fadiga & $\begin{array}{c}6,11 \\
(5,8)\end{array}$ & $\begin{array}{l}7,43 \\
(5,7)\end{array}$ & $\begin{array}{c}10,07 \\
(6,1)\end{array}$ & $\begin{array}{l}8,06 \\
(6,1)\end{array}$ & $\begin{array}{l}7,68 \\
(5,0)\end{array}$ & $\begin{array}{l}9,01 \\
(4,6)\end{array}$ & $\begin{array}{c}11,68 \\
(6,6)\end{array}$ & $\begin{array}{l}7,06 \\
(5,3)\end{array}$ & $\begin{array}{c}7,41 \\
(5,5)\end{array}$ \\
\hline Sonolência & $\begin{array}{l}7,39 \\
(6,3)\end{array}$ & $\begin{array}{l}8,10 \\
(5,8)\end{array}$ & $\begin{array}{c}10,24 \\
(6,2)\end{array}$ & $\begin{array}{l}9,59 \\
(6,1)\end{array}$ & $\begin{array}{l}8,36 \\
(5,3)\end{array}$ & $\begin{array}{l}8,85 \\
(4,5)\end{array}$ & $\begin{array}{c}12,74 \\
(6,4)\end{array}$ & $\begin{array}{l}8,06 \\
(5,7)\end{array}$ & $\begin{array}{l}7,65 \\
(5,0)\end{array}$ \\
\hline Atenção & $\begin{array}{c}10,37 \\
(6,6)\end{array}$ & $\begin{array}{l}11,24 \\
(4,7)\end{array}$ & $\begin{array}{c}10,02 \\
(5,5)\end{array}$ & $\begin{array}{l}9,69 \\
(5,3)\end{array}$ & $\begin{array}{l}11,29 \\
(4,3)\end{array}$ & $\begin{array}{l}11,41 \\
(3,9)\end{array}$ & $\begin{array}{l}6,62 \\
(5,5)\end{array}$ & $\begin{array}{l}13,03 \\
(4,5)\end{array}$ & $\begin{array}{c}12,74 \\
(4,4)\end{array}$ \\
\hline Estado de Alerta & $\begin{array}{l}6,44 \\
(5,7)\end{array}$ & $\begin{array}{c}12,78 \\
(5,0)\end{array}$ & $\begin{array}{c}12,92 \\
(4,6)\end{array}$ & $\begin{array}{l}9,24 \\
(5,3)\end{array}$ & $\begin{array}{c}11,38 \\
(4,5)\end{array}$ & $\begin{array}{c}11,25 \\
(4,4)\end{array}$ & $\begin{array}{l}6,44 \\
(5,7)\end{array}$ & $\begin{array}{c}12,78 \\
(4,9)\end{array}$ & $\begin{array}{c}12,92 \\
(4,6)\end{array}$ \\
\hline
\end{tabular}


Em relação aos sexos dos participantes e o padrão do cronotipo a ANOVA two way apresentou os seguintes resultados. Houve diferença durante os dias úteis e não úteis da semana de forma que as mulheres apresentaram diferenças significativas para a hora de acordar $[F(2,73)=$ $19,86 ; p<0,05]$ e para hora de deitar $[F(2,74)$ $=9,06 ; p<0,05]$ nos finais de semana e também para hora de acordar durante os dias úteis
$[F(2,72)=7,46 ; p<0,05]$. Os dados mostraram que mulheres MV dormem e acordam mais tarde que mulheres I e MM $(p<0,05)$. Em relação ao sexo masculino, os dados indicaram que existe diferença significativa para a hora de dei$\operatorname{tar}[F(2,29)=3,34 ; p<0,05]$ e hora de acordar $[F(2,28)=7,31 ; p<0,05]$ apenas durante nos finais de semana. Os escores do diário do sono dos sujeitos podem ser observados na Tabela 4 .

Tabela 4

Padrões Comportamentais do Sono de acordo com o Diário do Sono para Mulheres e Homens nos Dias da Semana e Finais de Semana

\begin{tabular}{|c|c|c|c|c|c|c|}
\hline \multirow{3}{*}{$\begin{array}{ll} & \text { Mulheres } \\
\text { Semana } & \end{array}$} & \multicolumn{4}{|c|}{ Diário do Sono } & & \\
\hline & \multicolumn{2}{|c|}{$\begin{array}{c}\text { Moderadamente } \\
\text { Matutino }\end{array}$} & \multicolumn{2}{|c|}{ Intermediário } & \multicolumn{2}{|c|}{$\begin{array}{l}\text { Moderadamente } \\
\text { Vespertino }\end{array}$} \\
\hline & Média & $D P$ & Média & $D P$ & Média & $D P$ \\
\hline Hora Dormir & $23: 11$ & \pm 82 & $23: 40$ & \pm 89 & $00: 36$ & \pm 114 \\
\hline Latência de Deitar & 10 & \pm 10 & 12 & \pm 19 & 12 & \pm 16 \\
\hline Hora Acordar & $06: 27$ & \pm 97 & $06: 58$ & \pm 105 & $07: 49$ & \pm 111 \\
\hline Latência Acordar & 13 & \pm 18 & 11 & 12 & 13 & \pm 18 \\
\hline \multicolumn{7}{|l|}{ Fim de semana } \\
\hline Hora Dormir & $23: 46$ & \pm 120 & $00: 21$ & \pm 88 & $01: 28$ & \pm 119 \\
\hline Latência de Deitar & 9 & \pm 8 & 13 & \pm 18 & 13 & \pm 19 \\
\hline Hora Acordar & 07:29 & \pm 112 & $08: 19$ & \pm 91 & 09:40 & \pm 101 \\
\hline Latência Acordar & 17 & \pm 15 & 16 & \pm 19 & 17 & \pm 15 \\
\hline Homens & \multicolumn{2}{|c|}{$\begin{array}{c}\text { Moderadamente } \\
\text { Matutino }\end{array}$} & \multicolumn{2}{|c|}{ Intermediário } & \multicolumn{2}{|c|}{$\begin{array}{l}\text { Moderadamente } \\
\text { Vespertino }\end{array}$} \\
\hline Semana & Média & $D P$ & Média & $D P$ & Média & $D P$ \\
\hline Hora Dormir & $23: 21$ & \pm 75 & $00: 10$ & \pm 69 & 01:00 & \pm 150 \\
\hline Latência de Deitar & 13 & \pm 11 & 13 & \pm 10 & 18 & \pm 15 \\
\hline Hora Acordar & $06: 26$ & \pm 52 & $07: 40$ & \pm 95 & $07: 58$ & \pm 117 \\
\hline Latência Acordar & 13 & \pm 14 & 10 & \pm 8 & 16 & \pm 16 \\
\hline \multicolumn{7}{|l|}{ Fim de Semana } \\
\hline Hora Dormir & $23: 50$ & \pm 89 & $00: 20$ & \pm 75 & $01: 37$ & \pm 135 \\
\hline Latência de Deitar & 13 & \pm 14 & 11 & \pm 8 & 28 & \pm 40 \\
\hline Hora Acordar & $07: 33$ & \pm 83 & $08: 18$ & \pm 97 & $09: 38$ & \pm 135 \\
\hline Latência Acordar & 26 & \pm 30 & 11 & \pm 7 & 19 & \pm 17 \\
\hline
\end{tabular}


Tabela 5

Escores da Escala Analógica Visual de acordo com o Cronotipo de Mulheres e Homens e o Horário de Medida

\begin{tabular}{|c|c|c|c|c|c|c|c|c|c|}
\hline \multirow{2}{*}{$\begin{array}{l}\text { Mulheres } \\
\text { Semana }\end{array}$} & \multicolumn{3}{|c|}{$\begin{array}{c}\text { Moderadamente } \\
\text { Matutino }\end{array}$} & \multicolumn{3}{|c|}{ Intermediário } & \multicolumn{3}{|c|}{$\begin{array}{c}\text { Moderadamente } \\
\text { Vespertino }\end{array}$} \\
\hline & $9 \mathrm{~h}$ & $15 \mathrm{~h}$ & $21 \mathrm{~h}$ & $9 \mathrm{~h}$ & $15 \mathrm{~h}$ & $21 \mathrm{~h}$ & $9 \mathrm{~h}$ & $15 \mathrm{~h}$ & $21 \mathrm{~h}$ \\
\hline Fadiga & $\begin{array}{l}5,78 \\
(5,2)\end{array}$ & $\begin{array}{l}7,87 \\
(5,4)\end{array}$ & $\begin{array}{l}10,81 \\
(5,9)\end{array}$ & $\begin{array}{l}7,07 \\
(5,3)\end{array}$ & $\begin{array}{l}8,57 \\
(4,7)\end{array}$ & $\begin{array}{l}10,67 \\
(5,3)\end{array}$ & $\begin{array}{l}10,14 \\
(5,8)\end{array}$ & $\begin{array}{l}8,64 \\
(5,6)\end{array}$ & $\begin{array}{l}8,58 \\
(5,9)\end{array}$ \\
\hline Sonolência & $\begin{array}{l}6,19 \\
(5,2)\end{array}$ & $\begin{array}{l}7,22 \\
(5,3)\end{array}$ & $\begin{array}{l}10,91 \\
(6,0)\end{array}$ & $\begin{array}{l}8,11 \\
(5,4)\end{array}$ & $\begin{array}{l}8,93 \\
(4,9)\end{array}$ & $\begin{array}{l}10,71 \\
(5,1)\end{array}$ & $\begin{array}{l}11,62 \\
(5,2)\end{array}$ & $\begin{array}{l}10,14 \\
(5,5)\end{array}$ & $\begin{array}{l}9,28 \\
(5,7)\end{array}$ \\
\hline Atenção & $\begin{array}{l}11,94 \\
(5,5)\end{array}$ & $\begin{array}{l}11,32 \\
(5,2)\end{array}$ & $\begin{array}{l}9,34 \\
(5,6)\end{array}$ & $\begin{array}{l}10,90 \\
(4,9)\end{array}$ & $\begin{array}{l}11,40 \\
(4,4)\end{array}$ & $\begin{array}{l}10,53 \\
(4,5)\end{array}$ & $\begin{array}{l}9,14 \\
(5,0)\end{array}$ & $\begin{array}{l}11,5 \\
(4,6)\end{array}$ & $\begin{array}{l}12,12 \\
(4,5)\end{array}$ \\
\hline Estado de Alerta & $\begin{array}{l}10,50 \\
(6,2)\end{array}$ & $\begin{array}{l}10,25 \\
(6,6)\end{array}$ & $\begin{array}{l}7,94 \\
(5,9)\end{array}$ & $\begin{array}{l}10,89 \\
(5,3)\end{array}$ & $\begin{array}{l}11,53 \\
(4,9)\end{array}$ & $\begin{array}{l}10,34 \\
(4,9)\end{array}$ & $\begin{array}{l}9,39 \\
(4,9)\end{array}$ & $\begin{array}{l}11,61 \\
(4,5)\end{array}$ & $\begin{array}{l}12,10 \\
(4,7)\end{array}$ \\
\hline \multicolumn{10}{|l|}{ Fim de Semana } \\
\hline Fadiga & $\begin{array}{l}7,03 \\
(5,9)\end{array}$ & $\begin{array}{l}7,81 \\
(6,5)\end{array}$ & $\begin{array}{l}10,44 \\
(6,4)\end{array}$ & $\begin{array}{l}7,49 \\
(6,1)\end{array}$ & $\begin{array}{l}7,40 \\
(4,9)\end{array}$ & $\begin{array}{l}9,19 \\
(4,3)\end{array}$ & $\begin{array}{l}10,67 \\
(6,6)\end{array}$ & $\begin{array}{l}7,44 \\
(5,3)\end{array}$ & $\begin{array}{l}7,60 \\
(5,5)\end{array}$ \\
\hline Sonolência & $\begin{array}{l}8,81 \\
(6,3)\end{array}$ & $\begin{array}{l}8,20 \\
(6,3)\end{array}$ & $\begin{array}{l}10,22 \\
(6,6)\end{array}$ & $\begin{array}{l}9,31 \\
(6,2)\end{array}$ & $\begin{array}{l}8,46 \\
(5,4)\end{array}$ & $\begin{array}{l}8,78 \\
(4,4)\end{array}$ & $\begin{array}{l}12,15 \\
(6,5)\end{array}$ & $\begin{array}{l}7,50 \\
(5,9)\end{array}$ & $\begin{array}{l}7,34 \\
(5,1)\end{array}$ \\
\hline Atenção & $\begin{array}{l}9,60 \\
(6,9)\end{array}$ & $\begin{array}{c}10,88 \\
(4,8)\end{array}$ & $\begin{array}{l}9,19 \\
(5,7)\end{array}$ & $\begin{array}{l}9,58 \\
(5,3)\end{array}$ & $\begin{array}{l}11,35 \\
(4,5)\end{array}$ & $\begin{array}{l}11,14 \\
(3,8)\end{array}$ & $\begin{array}{l}6,84 \\
(5,6)\end{array}$ & $\begin{array}{l}12,34 \\
(4,6)\end{array}$ & $\begin{array}{l}12,12 \\
(4,5)\end{array}$ \\
\hline Estado de Alerta & $\begin{array}{l}9,38 \\
(6,6)\end{array}$ & $\begin{array}{l}9,88 \\
(5,8)\end{array}$ & $\begin{array}{l}8,03 \\
(6,2)\end{array}$ & $\begin{array}{l}9,05 \\
(5,3)\end{array}$ & $\begin{array}{l}11,51 \\
(4,6)\end{array}$ & $\begin{array}{l}11,00 \\
(4,4)\end{array}$ & $\begin{array}{l}6,67 \\
(5,7)\end{array}$ & $\begin{array}{l}12,58 \\
(4,9)\end{array}$ & $\begin{array}{l}12,84 \\
(4,5)\end{array}$ \\
\hline Homens & \multicolumn{3}{|c|}{$\begin{array}{l}\text { Moderadamente } \\
\text { Matutino }\end{array}$} & \multicolumn{3}{|c|}{ Intermediário } & \multicolumn{3}{|c|}{$\begin{array}{l}\text { Moderadamente } \\
\text { Vespertino }\end{array}$} \\
\hline Semana & $9 \mathrm{~h}$ & $15 \mathrm{~h}$ & $21 \mathrm{~h}$ & $9 \mathrm{~h}$ & $15 \mathrm{~h}$ & $21 \mathrm{~h}$ & $9 \mathrm{~h}$ & $15 \mathrm{~h}$ & $21 \mathrm{~h}$ \\
\hline Fadiga & $\begin{array}{l}3,36 \\
(3,3)\end{array}$ & $\begin{array}{l}6,69 \\
(4,4)\end{array}$ & $\begin{array}{l}10,01 \\
(5,3)\end{array}$ & $\begin{array}{l}8,09 \\
(5,3)\end{array}$ & $\begin{array}{l}7,60 \\
(4,5)\end{array}$ & $\begin{array}{l}8,42 \\
(5,2)\end{array}$ & $\begin{array}{l}10,75 \\
(5,6)\end{array}$ & $\begin{array}{l}9,76 \\
(6,1)\end{array}$ & $\begin{array}{l}8,83 \\
(5,6)\end{array}$ \\
\hline Sonolência & $\begin{array}{l}4,07 \\
(3,5)\end{array}$ & $\begin{array}{l}7,49 \\
(5,2)\end{array}$ & $\begin{array}{l}11,50 \\
(6,7)\end{array}$ & $\begin{array}{l}8,33 \\
(5,3)\end{array}$ & $\begin{array}{l}8,15 \\
(4,9)\end{array}$ & $\begin{array}{l}8,81 \\
(5,0)\end{array}$ & $\begin{array}{l}12,31 \\
(5,6)\end{array}$ & $\begin{array}{c}10,56 \\
(5,9)\end{array}$ & $\begin{array}{l}10,04 \\
(5,9)\end{array}$ \\
\hline Atenção & $\begin{array}{l}12,29 \\
(6,1)\end{array}$ & $\begin{array}{l}11,07 \\
(5,2)\end{array}$ & $\begin{array}{c}10,86 \\
(5,8)\end{array}$ & $\begin{array}{c}10,39 \\
(4,8)\end{array}$ & $\begin{array}{l}11,62 \\
(3,6)\end{array}$ & $\begin{array}{l}11,89 \\
(4,5)\end{array}$ & $\begin{array}{l}9,80 \\
(5,3)\end{array}$ & $\begin{array}{l}12,23 \\
(5,1)\end{array}$ & $\begin{array}{c}12,64 \\
(3,7)\end{array}$ \\
\hline Estado de Alerta & $\begin{array}{l}11,07 \\
(6,8)\end{array}$ & $\begin{array}{l}11,00 \\
(5,5)\end{array}$ & $\begin{array}{l}11,40 \\
(6,1)\end{array}$ & $\begin{array}{l}10,27 \\
(4,7)\end{array}$ & $\begin{array}{l}11,52 \\
(4,2)\end{array}$ & $\begin{array}{l}11,35 \\
(4,7)\end{array}$ & $\begin{array}{l}8,72 \\
(5,4)\end{array}$ & $\begin{array}{c}10,99 \\
(5,7)\end{array}$ & $\begin{array}{l}12,69 \\
(3,9)\end{array}$ \\
\hline \multicolumn{10}{|l|}{ Fim de Semana } \\
\hline Fadiga & $\begin{array}{l}3,86 \\
(4,4)\end{array}$ & $\begin{array}{l}6,43 \\
(3,3)\end{array}$ & $\begin{array}{l}8,71 \\
(5,6)\end{array}$ & $\begin{array}{l}9,71 \\
(5,9)\end{array}$ & $\begin{array}{l}8,50 \\
(5,0)\end{array}$ & $\begin{array}{l}8,35 \\
(5,1)\end{array}$ & $\begin{array}{c}12,36 \\
(6,7)\end{array}$ & $\begin{array}{l}6,21 \\
(5,3)\end{array}$ & $\begin{array}{l}6,68 \\
(5,5)\end{array}$ \\
\hline Sonolência & $\begin{array}{l}4,29 \\
(4,9)\end{array}$ & $\begin{array}{l}6,62 \\
(4,9)\end{array}$ & $\begin{array}{l}9,86 \\
(6,2)\end{array}$ & $\begin{array}{c}10,38 \\
(5,6)\end{array}$ & $\begin{array}{l}8,19 \\
(5,3)\end{array}$ & $\begin{array}{l}8,81 \\
(4,8)\end{array}$ & $\begin{array}{c}13,92 \\
(6,1)\end{array}$ & $\begin{array}{l}8,00 \\
(5,6)\end{array}$ & $\begin{array}{l}7,49 \\
(5,4)\end{array}$ \\
\hline Atenção & $\begin{array}{l}11,21 \\
(6,6)\end{array}$ & $\begin{array}{l}11,29 \\
(4,1)\end{array}$ & $\begin{array}{l}13,00 \\
(3,9)\end{array}$ & $\begin{array}{l}9,96 \\
(4,5)\end{array}$ & $\begin{array}{l}11,12 \\
(4,3)\end{array}$ & $\begin{array}{l}12,23 \\
(4,3)\end{array}$ & $\begin{array}{l}5,91 \\
(5,9)\end{array}$ & $\begin{array}{c}14,26 \\
(4,1)\end{array}$ & $\begin{array}{l}13,62 \\
(3,9)\end{array}$ \\
\hline Estado de Alerta & $\begin{array}{c}10,50 \\
(6,6)\end{array}$ & $\begin{array}{c}10,86 \\
(4,3)\end{array}$ & $\begin{array}{c}12,14 \\
(4,3)\end{array}$ & $\begin{array}{l}9,77 \\
(5,4)\end{array}$ & $\begin{array}{c}11,19 \\
(4,4)\end{array}$ & $\begin{array}{c}11,96 \\
(4,5)\end{array}$ & $\begin{array}{l}5,41 \\
(5,9)\end{array}$ & $\begin{array}{c}12,92 \\
(5,2)\end{array}$ & $\begin{array}{c}12,91 \\
(4,9)\end{array}$ \\
\hline
\end{tabular}


Os resultados também apontaram diferenças significativas entre os cronotipos de mulheres e homens e as dimensões da escala analógica visual (Tabela 5). Os dados mostram que mulheres apresentaram diferenças no período das $21 \mathrm{~h}$ para dimensão fadiga $[F(2,39)=3,26 ; p<0,05]$ e às $15 \mathrm{~h}$ para as dimensões atenção $[F(2,39)$ $=5,94 ; p<0,05$; e estado de alerta $[F(2,39)=$ $8,58 ; p<0,05]$. A análise post hoc Turkey mostrou que mulheres MM apresentam maior fadiga que mulheres com I $(p<0,05)$. Além disso, as mulheres MV apresentam maiores níveis de atenção e estado de alerta às 15 horas quando comparado com mulheres MM $(p<0,05)$. A análise entre os homens mostrou diferença significativa apenas para dimensão de estado de alerta $[F(2,39)=5,51 ; p<0,05]$, onde os sujeitos I apresentaram maiores escores de alerta que os sujeitos MV $(p<0,05)$. De forma geral, as mulheres sofrem maior variação nas suas dimensões comportamentais quando relacionadas aos homens $(p<0,01)$.

\section{Dimensões Comportamentais do Sono e Traços de Personalidade}

A Figura 1 mostra que todos os sujeitos apresentam maior escore para o traço de personalidade amabilidade e menor escore para neuroticismo. A análise multivariada de variância (MANOVA) mostrou diferença significativa entre os cronotipos e os traços de personalidade $\left[\lambda=0,78 ; F(10,198)=2,52 ; p<0,05 ; \eta^{2}=0,11\right]$. Observou-se diferença significativa apenas para os traços de extroversão $[F(2,103)=3,65 ; p<$ 0,$\left.05 ; \eta^{2}=0,06\right]$ e amabilidade $[F(2,103)=8,03$; $\left.p<0,05 ; \eta^{2}=0,14\right]$. O teste post hoc Bonferroni indicou que sujeitos com cronotipo I apresentaram maiores escores nos traços extroversão e amabilidade do que indivíduos MV $(p<0,05)$. Além disso, os dados mostram que sujeitos MM apresentam maiores escores no traço amabilidade que sujeitos MV $(p<0,05)$. Contudo, não se verificou alterações entre os dados quando foram controladas as covariáveis: hora de acordar e hora de dormir nos dias úteis e não úteis da semana, qualidade de sono e as dimensões da escala analógica visual $(p>0,05)$.

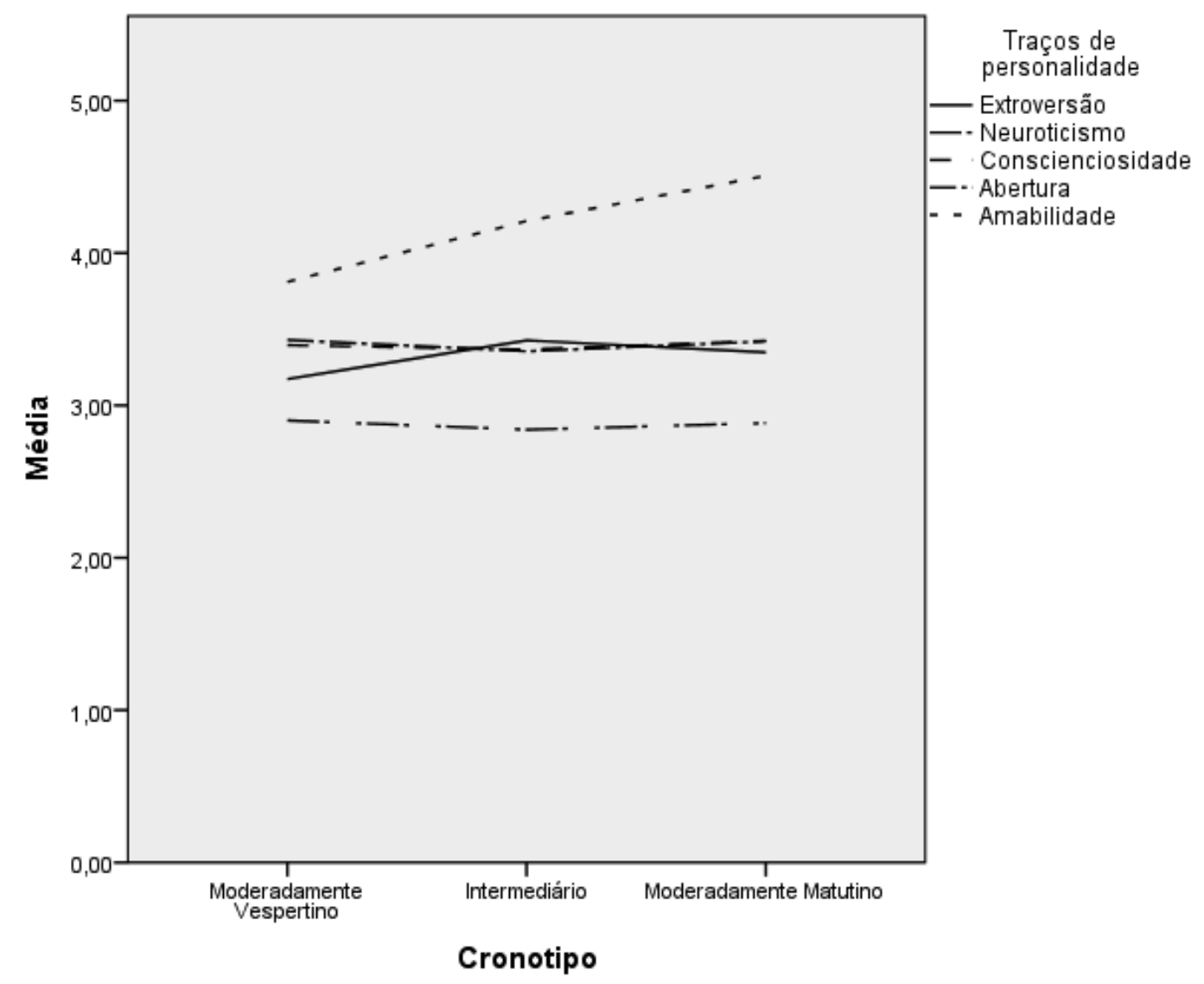

Figura 1. Valores de traços de personalidade de acordo com o cronotipo. 
Também foi realizado uma MANOVA nos dados de acordo com o sexo dos participantes (Figura 2). Mulheres e homens apresentaram maiores escores para o fator amabilidade e menores escores para o fator neuroticismo, independentemente do cronotipo. A análise não apresentou diferença entre os cronotipos e os traços de personalidade para os homens, porém observaram-se diferenças entre as mulheres $[\lambda=$ 0,$\left.74 ; F(10,134) ; p<0,005 ; \eta^{2}=0,14\right]$ em relação ao traço amabilidade $[F(2,71)=7,74 ; p<0,05$; $\left.\eta^{2}=0,18\right]$. Mulheres MM apresentaram maiores escores em amabilidade que mulheres MV.
A

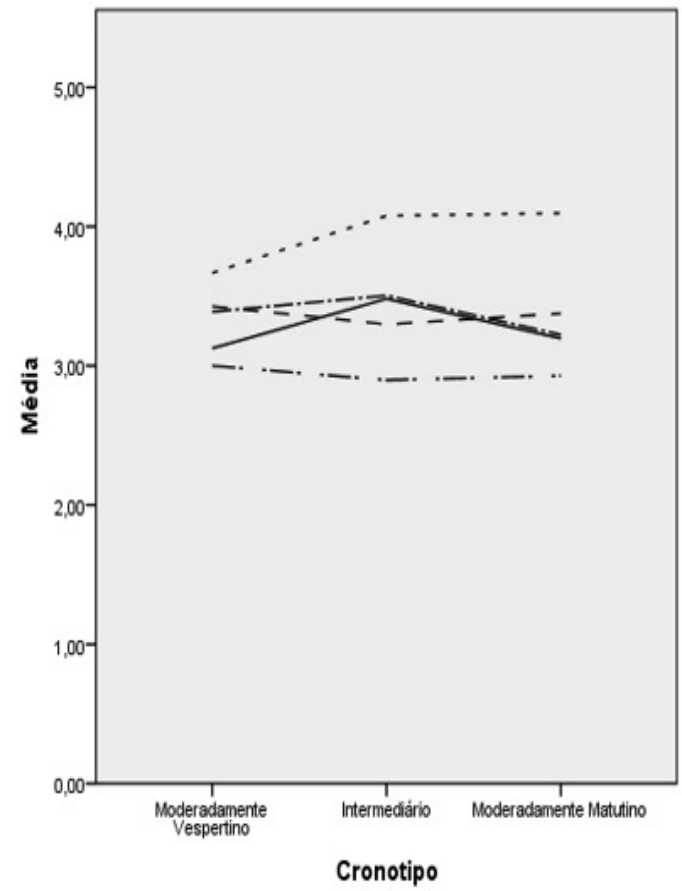

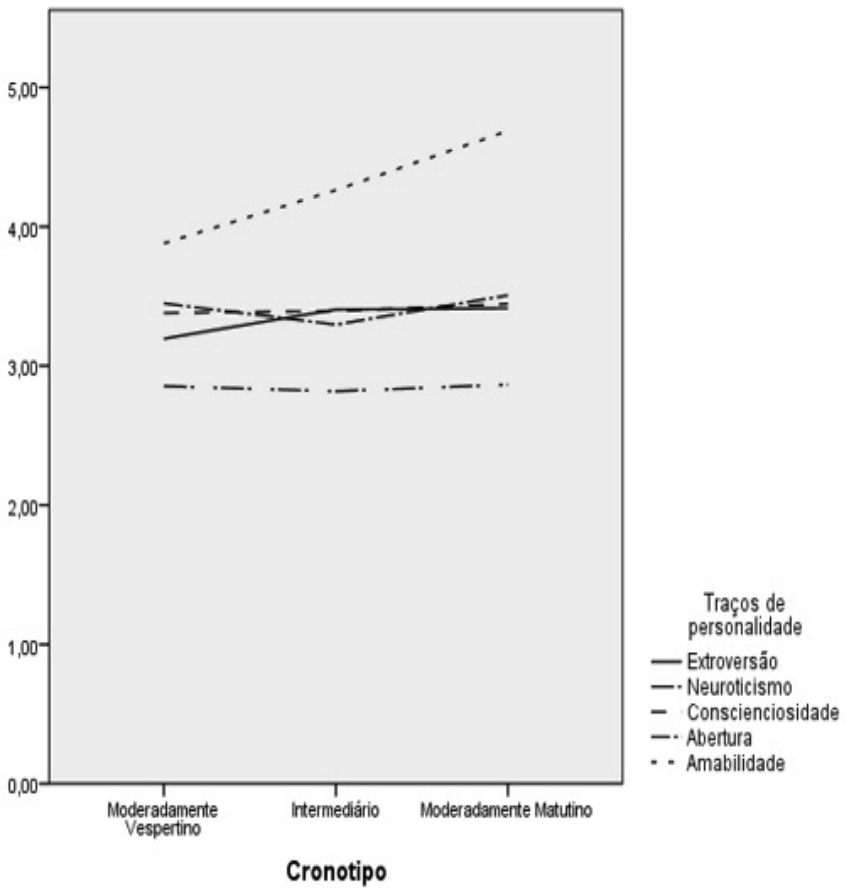

Figura 2. Valores de traços de personalidade de acordo com o cronotipo e o sexo: A(Homens) e B (Mulheres).

A análise dos dados não mostrou diferença significativa entre os traços de personalidade e cronotipos de mulheres e homens quando controlado os padrões comportamentais do sono, as dimensões de atenção, estado de alerta, fadiga, tensão e qualidade de sono dos participantes ( $p$ $>0,05)$. Pode-se verificar, ainda, que não houve diferença entre os traços de personalidade e o padrão de cronotipo entre os sexos, ou seja, homens e mulheres MM $(p=0,46)$, I $(p=0,40)$ e MV $(p=0,83)$.

\section{Discussão}

O objetivo deste estudo foi verificar a influência dos padrões comportamentais do sono nos traços de personalidade de homens e mulheres tendo como base o Modelo dos Cinco Grandes Fatores de Personalidade, ou seja, verificar a influência do cronotipo na análise de termos da linguagem que sujeitos usam para descrever a si próprio e os outros em seu ambiente (Jonh \& Srivastava, 1999).

Conforme os critérios da análise na cronobiologia verificaram-se características temporais do ciclo vigília-sono envolvidas na estrutura do tempo biológico dos participantes da pesquisa, como por exemplo, o cronotipo, características de qualidade de sono, uso de psicotrópicos, transtornos neurológicos e características ambientais (Blatter \& Cajochen, 2007; Portaluppi, Smolensky, \& Touitou, 2010). Os sujeitos apresentaram um padrão de cronotipo bem definido, sendo classificados em Moderadamente Matuti- 
no, Intermediário e Moderadamente Vespertino. É comum que maior parte da população apresente um cronotipo Intermediário, principalmente entre jovens adultos (Preckel, Lipnevich, Schneider, \& Roberts, 2011; Randler, 2007).

Ao verificar as diferenças individuais relacionadas aos cronotipos, percebeu-se que mesmo com durações de sono equivalentes, foi possível confirmar os resultados encontrados por Horne e Östberg (1975). Os indivíduos moderadamente matutinos (MM) foram aqueles que se sentem mais dispostos para realizar atividades no período da manhã, ao passo que os moderadamente vespertinos (MV) sentem-se mais dispostos durante a noite. Também foram identificadas diferenças significativas entre hora de deitar e hora de acordar dos cronotipos MM e MV.

Nota-se a influência de zeitgebers sociais na variação do comportamento de acordo com dias úteis e não úteis da semana. Conforme Delattre (2004), os zeitgebers referem-se a variações cíclicas de fatores ambientais capazes de influenciar a expressão rítmica. Como visto no presente estudo, preferências para realizar atividades diárias de acordo com hora e os dias da semana pode ser um sincronizador funcional de fatores da personalidade. Nessa perspectiva, os cronotipos são considerados prováveis dimensões da personalidade devido os padrões de sono variarem individualmente e possuírem estabilidade concreta, além de ser constituídos por fatores genéticos e exógenos (Randler et al., 2014).

É possível notar que sujeitos I e MM apresentaram maiores escores em amabilidade e extroversão que os sujeitos MV, respectivamente. Esse estudo descreveu que pessoas com diferentes traços de personalidades possuem horas especificas para realizar suas atividades e que existe uma relação com o padrão de preferência comportamental do sono (Cavallera \& Giudici, 2007; Díaz-Morales et al., 2007; Horne \& Östberg, 1977; Mecacci, Righi, \& Rocchetti, 2004; Mecacci et al., 1986; Neubauer, 1992; Schubert \& Randler, 2008; Tankova et al., 1994; Vollmer \& Randler, 2012). Ainda, os indivíduos com hábitos mais noturnos que apresentaram maiores escores em extroversão e amabilidade, traços que são caracterizados por comportamentos menos frios e indelicados, e com mais habilidades de adaptação às normas de um grupo a qual pertencem, mostraram-se mais ativos e entusiasmados.

Alguns estudos apontam a existência da correlação entre extroversão e vespertinidade, sugerindo que sujeitos que consomem mais cafeína tendem a manter um estilo de vida noturno ativo, e também que pessoas extrovertidas possuem níveis basais de estimulação mais baixos do que os introvertidos, o que explicaria a utilização de estimulantes a fim de atingir um nível de estimulação (Adan, Prat, Fabbri, \& Sànchez-Turet, 2008; Kerkhof, 1985; Mitchell \& Redman, 1993). Porém, o presente estudo indica que apesar do consumo ocasional de cafeína, nicotina e álcool da amostra, não foram observados associações entre os cronotipos e o consumo de tais substâncias. É possível que estas substâncias não resultem em graves alterações comportamentais nos traços de personalidade associado aos fatores do sono, independentemente do sexo dos participantes. Porém, faz-se necessária a condução de estudos psicofisiológicos de medidas de cortisol e melatonina que verifiquem os efeitos de estimulantes/inibitórios no sistema nervoso central associado aos padrões de sono e aos traços de personalidade (Bueno \& Way, 2012; Miguel, 2012; Owsley, Sekuler, \& Siemsen, 1983; Paine, Gander, \& Travier, 2006).

Neubauer (1992) ao comparar os mesmos fatores investigados no presente estudo, encontrou correlações baixas, porém significativas entre extroversão e neuroticismo com o cronotipo moderadamente vespertino, indicando que os sujeitos moderadamente vespertinos possuem características como entusiasmo, altivez, (exemplos de extroversão) e certa tendência a apresentar estados negativos (exemplo de neuroticismo). Em outros estudos a tendência de correlação entre vespertinidade e extroversão também apareceu como principal correlação (Horne \& Östberg, 1977; Hsu, Gau, Shang, Chiu, \& lee, 2012; Tankova et al., 1994). Assim, observa-se que mesmo apresentando certa diferença nos resultados, os estudos possuem uma diferença significativa entre os traços de extroversão e vespertinidade. 
No que se refere ao grau de Fadiga e Sonolência, tanto mulheres como homens MM mostraram-se mais dispostos no período da manhã e indispostos à noite, enquanto que os MV estavam mais cansados pela manhã do que à noite. $\mathrm{O}$ mesmo pode ser observado com relação ao Estado de Alerta e Atenção apenas entre as mulheres, pois ao comparar esses fatores entre os homens MV e MM, não houve grandes diferenças de médias. Dessa forma, ao confrontar os resultados obtidos neste estudo com os da literatura, nota-se que as mulheres exibem um padrão mais sensível ao estado de alerta e vigor (Adan \& Sánchez-Turet, 2001).

É importante destacar a relevância de replicações do presente estudo. No entanto, considerando-se que a variação ambiental da intensidade de luz é capaz de provocar variações em medidas neurocognitivas e psicológicas nos ritmos circadianos (Besílio et al., 2012; Blatter \& Cajochen, 2007; Marques \& Menna-Barreto, 1997; Pereira, Sabino, \& Umemura, 2012), é necessário caracterizar geograficamente a localização dos sujeitos da pesquisa, bem como suas características individuais como realizado neste estudo. Outro ponto importante é caracterizar os participantes quanto um maior $n$ amostral e variações psicológicas de estados emocionais.

\section{Considerações Finais}

Os padrões circadianos do sono são capazes de alterar medidas comportamentais e neurocognitivas, como por exemplo, respostas atencionais e mnemônicas. Contudo, é possível que as preferencias para realizar atividades diárias estejam associadas às dimensões de personalidade. $\mathrm{O}$ presente estudo encontrou diferenças nos traços de personalidade de homens e mulheres ao relacionado ao cronotipo. É importante considerar que todos os participantes desta pesquisa eram estudantes universitários (possuem mudanças nos padrões comportamentais sócias conforme as necessidades acadêmicas) e estão localizados geograficamente a " $07^{\circ} 07$ ' 10.19 ” S de latitude e longitude $34^{\circ} 50$ > 42.04» com uma amplitude de 43.02, ou seja, a densidade fótica é capaz de estar associado a estes mecanismos funcionais.

\section{Referências}

Adan, A., \& Sánchez-Turet, M. (2001). Gender differences in diurnal variations of subjective activation and mood. Chronobiology International, 18(3), 491-502. doi:10.1081/CBI-100103971

Adan, A., Prat, G., Fabbri, M., \& Sànchez-Turet, M. (2008). Early effects of caffeinated and decaffeinated coffee on subjective state and gender differences. Progress in Neuro-Psychopharmacology and Biological Psychiatry, 32(7), 16981703. doi:10.1016/j.pnpbp.2008.07.005

Andrade, J. M. (2008). Evidências de Validade do Inventário dos Cinco Grandes Fatores de Personalidade para o Brasil (Tese de doutorado, Universidade de Brasília, DF, Brasil).

Benedito-Silva, A. A., Menna-Barreto, L., Marques, N., \&Tenreiro, S. (1990). A self-assessment questionnaire for the determination of morningness-eveningness types in Brazil. Progress in Clinical and Biological Research, 341, 89-98.

Besílio, A. S., Carneiro, B. T., Silva, C. A., Fortes, F. S., \&Araújo, J. F. (2012). Métodos cronobiológicos aplicados à neurociência clínica e experimental. Métodos em Neurociências, 114-127.

Blatter, K., \& Cajochen, C. (2007). Circadian rhythms in cognitive performance: Methodological constraints, protocols, theoretical underpinnings. Physiology \& Behavior, 90(2), 196208. doi:10.1016/j.physbeh.2006.09.009

Bueno, C., \& Way, D. (2012). Gênese e ontogênese do ritmo de sono/vigília em humanos. Revista de Biologia, 9(3), 62-67. doi:10.7594/revbio.09.03.12

Cavallera, G. M., \& Giudici, S. (2007). Morningness and eveningness personality: A survey in literature from 1995 up till 2006. Personality and Individual Differences, 44(1), 3-21. doi:10.1016/j. paid.2007.07.009

Cunha, J. A. (1999). Estudo dos pontos de corte do BDI e do BAI na versão em Português [Resumo]. In VIII Congresso Nacional de Avaliação Psicológica (p. 78). Porto Alegre, RS.

Delattre, E. (2004). Ritmos hormonais do pâncreas endócrino: Dos fundamentos cronobiológicos às implicações clínicas. Medicina, 37, 51-64.

DeYoung, C. G., Hasher, L., Djikic, M., Criger, B., \& Peterson, J. B. (2007). Morning people are stable people: Circadian rhythm and the higher-order 
factors of the Big Five. Personality and Individual Differences, 43(2), 267-276. doi:10.1016/j. paid.2006.11.030

Díaz-Morales, J. F., de León, M. C. D., \& Sorroche, M. G. (2007). Validity of the morningness-eveningness scale for children among Spanish adolescents. Chronobiology International, 24(3), 435-447. doi:10.1080/07420520701420659

Duggan, K. A., Friedman, H. S., McDevitt, E. A., \& Mednick, S. A. (2014). Personality and Healthy Sleep: The Importance of Conscientiousness and Neuroticism. PLOSone, 9(3), 1-11.

Furlani, R., \& Ceolim, M. F. (2005). Padrões de sono de estudantes ingressantes na Graduação em Enfermagem. Revista Brasileira de Enfermagem, 58(3), 320-324. doi:10.1590/S003471672005000300013

Horne, J. A., \& Östberg, O. (1975). A self-assessment questionnaire to determine morningness-eveningness in human circadian rhythms. International Journal of Chronobiology, 4(2), 97-110.

Horne, J. A., \& Östberg, O. (1977). Individual differences in human circadian rhythms. Biological Psychology, 5(3), 179-190.

Hsu, C. Y., Gau, S. S. F., Shang, C. Y., Chiu, Y. N., \& Lee, M. B. (2012). Associations between chronotypes, psychopathology, and personality among incoming college students. Chronobiology International, 29(4), 491-501. doi:10.3109/0 7420528.2012 .668995

Jackson, L. A., \& Gerard, D. A. (1996). Diurnal types, the" Big Five" personality factors, and other personal characteristics. Journal of Social Behavior and Personality, 11(2), 273.

John, O. P., \& Srivastava, S. (1999). The Big Five trait taxonomy: History, measurement, and theoretical perspectives. Handbook of Personality: Theory and Research, 2(1999), 102-138.

Kerkhof, G. A. (1985). Inter-individual differences in the human circadian system: A review. Biological Psychology, 20(2), 83-112. doi:10.1016/0301-0511(85)90019-5

Laneiro, T., Brites, R., Tap, P., Silva, A., Reguinga, O., \& Guerra, S. (2011). A influência da alteração dos ciclos circadianos na auto-percepção individual: A experiência subjectiva de fadiga, atenção, tensão e satisfação na tarefa. Psicologia, Saúde \& Doenças, 12(1), 3-17.
Larsen, R. J. (1985). Individual differences in circadian activity rhythm and personality. Personality and Individual Differences, 6(3), 305-311. doi:10.1016/0191-8869(85)90054-6

Marques, N., \& Menna-Barreto, L. (1997). Cronobiologia: Princípios e Aplicacões: Vol. 12. São Paulo, SP: Editora da Universidade de São Paulo.

Mecacci, L., Righi, S., \& Rocchetti, G. (2004). Cognitive failures and circadian typology. Personality and Individual Differences, 37(1), 107-113. doi:10.1016/j.paid.2003.08.004

Mecacci, L., Zani, A., Rocchetti, G., \& Lucioli, R. (1986). The relationships between morningness-eveningness, ageing and personality. Personality and Individual Differences, 7(6), 911-913. doi:10.1016/0191-8869(86)90094-2

Miguel, M. A. L. (2012). Estimativa de tempo em humanos: Bases, ontogênese e variação diária. Revista de Biologia, 9(3), 74-79. doi:10.7594/ revbio.09.03.14

Mitchell, P. J., \& Redman, J. R. (1993). The relationship between morningness-eveningness, personality and habitual caffeine consumption. Personality and Individual Differences, 15(1), 105-108. doi:10.1016/0191-8869(93)90050-D

Neubauer, A. C. (1992). Psychometric comparison of two circadian rhythm questionnaires and their relationship with personality. Personality and Individual Differences, 13(2), 125-131. doi:10.1016/0191-8869(92)90035-N

Owsley, C., Sekuler, R., \& Siemsen, D. (1983). Contrast sensitivity throughout adulthood. Vision Research, 23, 689-699.

Paine, S. J., Gander, P. H., \& Travier, N. (2006). The epidemiology of morningness/eveningness: Influence of age, gender, ethnicity, and socioeconomic factors in adults (30-49 years). Journal of Biological Rhythms, 21(1), 68-76. doi:10.1177/0748730405283154

Pereira, D. S., Sabino, F. C., \& Umemura, G. S. (2012). Period3: Um gene relacionado com asincronização de ritmos circadianos pela luz. Revista de Biologia, 9(3), 26-31. doi:10.7594/ revbio.09.03.05

Portaluppi, F., Smolensky, M. H., \& Touitou, Y. (2010). Ethics and methods for biological rhythm research on animals and human beings. 
Chronobiology International, 27(9-10), 19111929. doi:10.3109/07420528.2010.516381

Preckel, F., Lipnevich, A. A., Schneider, S., \& Roberts, R. D. (2011). Chronotype, cognitive abilities, and academic achievement: A meta-analytic investigation. Learning and Individual Differences, 21(5), 483-492. doi:10.1016/j.lindif.2011.07.003

Randler, C. (2007). Gender differences in morningness-eveningness assessed by self-report questionnaires: A meta-analysis. Personality and Individual Differences, 43(7), 1667-1675. doi:10.1016/j.paid.2007.05.004

Randler, C., Baumann, V. P., \& Horzum, M. B. (2014). Morningness-eveningness, Big Five and the BIS/BAS inventory. Personality and Individual Differences, 66, 64-67. doi:10.1016/j. paid.2014.03.010

Randler, C., \& Saliger,L.(2011). Relationship between morningness-eveningness and temperament and character dimensions in adolescents. Personality and Individual Differences, 50(2), 148-152. doi:10.1016/j.paid.2010.09.016

Roenneberg, T., Kuehnle, T., Pramstaller, P. P., Ricken, J., Havel, M., Guth, A., \& Merrow, M. (2004). A marker for the end of adolescence. Current Biology, 14(24), 1038. doi:10.1016/j. cub.2004.11.039

Russo, P. M., Leone, L., Penolazzi, B., \& Natale, V. (2012). Circadian preference and the big five: The role of impulsivity and sensation seeking. Chronobiology International, 29(8), 1121-1126. doi:10.3109/07420528.2012.706768

Schubert, E., \& Randler, C. (2008). Association between chronotype and the constructs of the Three-Factor-Eating-Questionnaire. Appetite, 51(3), 501-505. doi:10.1016/j.appet.2008.03.018
Tankova, I., Adan, A., \& Buela-Casal, G. (1994). Circadian typology and individual differences. A review. Personality and Individual Differences, 16(5), 671-684. doi:10.1016/01918869(94)90209-7

Tonetti, L., Adan, A., Caci, H., De Pascalis, V., Fabbri, M., \& Natale, V. (2010). Morningnesseveningness preference and sensation seeking. European Psychiatry, 25(2), 111-115. doi:10.1016/j.eurpsy.2009.09.007

Torsvall, L., \& Åkerstedt, T. (1980). A diurnal type scale: Construction, consistency and validation in shift work. Scandinavian Journal of Work, Environment \& Health, 283-290. doi:10.5271/ sjweh.2608

Valdez, P., Ramirez, C., \& Garcia, A. (2012). Circadian rhythms in cognitive performance: Implications for neuropsychological assessment. Chrono Physiology and Terapy, 2, 81-82. doi:http:// dx.doi.org/10.2147/CPT.S32586

Vollmer, C., \& Randler, C. (2012). Circadian preferences and personality values: Morning types prefer social values, evening types prefer individual values. Personality and Individual Differences, 52(6), 738-743. doi:10.1016/j. paid.2012.01.001
Recebido: 03/07/2016

$1^{a}$ revisão: $26 / 12 / 2016$

Aceite final: $21 / 02 / 2017$

(C) O(s) autor(es), 2018. Acesso aberto. Este artigo está distribuído nos termos da Licença Internacional Creative Commons Atribuição 4.0 (http://creativecommons.org/licenses/by/4.0/), que permite o uso, distribuição e reprodução sem restrições em qualquer meio, desde que você dê crédito apropriado ao(s) autor(es) original(ais) e à fonte, fornecer um link para a licença Creative Commons e indicar se as alterações foram feitas. 